\title{
Sentiment de lassitude et changements de mobilier domestique
}

\author{
Femmes tiraillées entre pulsions \\ socioculturelles, tendances à la surconsommation et \\ soucis écologiques
}

Salma Zouaghi

\begin{abstract}
[Résumé] Le marché d'ameublement français réunit 6.220 établissements et 55.100 salariés ${ }^{1}$. En 2011, il a enregistré son plus haut niveau depuis 1988 avec un chiffre d'affaires atteignant 9,83 milliards d'euros $\mathrm{TTC}^{2}$. Ces chiffres témoignent de son importance et font émerger diverses questions auprès des scientifiques et des sociologues notamment sur le thème de la consommation. Tel est le cas de cette présente recherche qui étudie les phénomènes de lassitude par rapport à l'usage durable du mobilier domestique. Elle se fonde sur une enquête quantitative réalisée en France auprès de 126 participants ${ }^{3}$. Les résultats montrent, d'une part, que la consommation de mobilier implique principalement le genre féminin et permettent de distinguer, d'autre part, deux situations différentes entre les femmes âgées de 25 à 35 ans et les femmes âgées de 45 à 65 ans. La première situation montre que la majorité des jeunes femmes (25/35 ans) sont tiraillées entre deux phénomènes. D'un côté, le sentiment fréquent de routine et de lassitude qui stimule le désir de changement et d'innovation. D'un autre côté, le faible pouvoir d'achat qui empêche de mettre fin à ce malaise. La deuxième situation établit que la majorité des femmes plus âgées (45/65 ans) sont moins impliquées dans ce phénomène de lassitude et de désir de consommation et plus satisfaites de la durabilité de leurs aménagements intérieurs. Cependant, une minorité de cette catégorie adhère à l'activité du changement pour refléter une image plus jeune, plus tendance. Et ceci est possible, dans la plupart des cas, grâce à un pouvoir d'achat plus confortable que celui des jeunes femmes. Dans les deux catégories, en dépit de la tension exercée par des facteurs socioculturels, économiques, médiatiques, etc., la majorité des femmes de l'échantillon a développé un comportement de consommation adapté à leurs différentes situations avec des tendances en faveur de l'écologie.

Mots-clés : Mobilier domestique, sentiment de lassitude, femmes, consommation, changement, écologie.
\end{abstract}

\footnotetext{
${ }^{1}$ Source : UNEDIC 2010. Information disponible sur le site:

http://www.fnaem.fr/dl/communiques/FNAEM_COMMUNIQUE_94.pdf

${ }^{2}$ Source : http://www.fnaem.fr/dl/communiques/FNAEM_COMMUNIQUE_113.pdf

${ }^{3}$ Etude appliquée sur un échantillon de 126 français, habitants de la région parisienne et lyonnaise.
} 


\section{Introduction}

Ce projet de recherche est né d'un doute personnel engendré par ma propre implication dans un phénomène qui prend une tournure cyclique. Il s'agit du sentiment de lassitude et de malaise vis-à-vis de l'usage durable des mêmes mobiliers domestiques. Ce sentiment me pousse à désirer des changements afin de renouveler l'ambiance globale. Ceci m'offre la possibilité de vivre confortablement dans un cadre qui produit perpétuellement le plaisir du changement ou encore le plaisir du neuf. Suite à chacun de ces changements, je me sens, dans l'instant, gagnée par une sensation de bien-être, mais qui est bien éphémère. En effet, peu à peu pointe une sensation de malaise qui perturbe mon contentement. Celle-ci, causée par la monotonie et la routine, survient à la fin d'une période d'usage, dont la durée tend à s'écourter. Ma conduite est devenue source de réflexion et d'interrogations, notamment sur la question de l'augmentation de la fréquence du besoin du changement. Je me suis alors intéressée aux raisons expliquant ce sentiment d'ennui qui est de plus en plus récurrent, alors que plusieurs générations antérieures ont réussi à vivre dans le même environnement aménagé avec les mêmes meubles durant toute une vie. Comment ce sentiment se manifeste-t-il ? Que cela pourrait-il impliquer sur le comportement de consommation et sur l'environnement ? Dans cette étude, les axes de recherche reposent sur trois questions principales :

- Quelle est la catégorie de personnes les plus touchées par le sentiment de lassitude quant à la durabilité des aménagements intérieurs : les femmes ou les hommes ? Les jeunes ou les plus âgés?

- Comment cette catégorie de personnes réagit-elle en réponse à ce sentiment de lassitude?

- Quelles sont les préoccupations et mobilisations écologiques de cette catégorie?

\section{Le secteur de la consommation de mobilier domestique en France}

L'acte de consommer, s'il a toujours existé, a particulièrement été exacerbé depuis la Seconde Guerre mondiale dans nos sociétés. En rendant les produits et les services offerts payants, les entreprises marchandes ont participé à rendre notre vie quotidienne source de consommation permanente. En effet, en tant qu'individu, nous sommes toujours en train de consommer : nourriture, objets matériels, idées ou encore services. La consommation a produit du bonheur et du bien-être et a favorisé l'enrichissement de certains pays. En conséquence, elle est non seulement au centre des préoccupations des économistes, des politiciens, des ingénieurs, des chimistes, etc., mais aussi des chercheurs scientifiques. Ceux-ci tentent de comprendre son mécanisme, ses fondements, les acteurs et les facteurs qu'elle implique ou encore ses conséquences sur l'homme, la société et la planète. 
Consommer semble être, tout d'abord, un réel bonheur. Les êtres humains sont toujours en quête de bonheur pour affirmer leur existence. Ils consomment alors, autant que leurs moyens le leur permettent, et très souvent, au-delà de leurs propres besoins. Comme le signale Jean Baudrillard dans La société de consommation (1970 : 49) : «Toutes les sociétés ont toujours gaspillé, dilapidé, dépensé et consommé au-delà du strict nécessaire, pour la simple raison que c'est dans la consommation d'un excédent, d'un superflu que l'individu comme la société se sentent non seulement exister mais vivre. » Pour assurer » la pérennité » de la consommation et la réussite économique, il est important de créer perpétuellement des besoins chez le consommateur en l'informant, l'influençant et pourquoi pas en le « manipulant ». Pour y parvenir, de nombreuses recherches, depuis les années 60, ont tenté de comprendre la psychosociologie des individus afin de pouvoir orienter leurs besoins et leurs désirs. L'objectif visé par ces recherches étant d'organiser, pour le consommateur, les conditions et l'accessibilité à la fois géographique et financière ainsi que des prétextes qui justifient son acte d'achat ${ }^{4}$. Le marketing, les publicités, le packaging susciteront l'attention du consommateur et participeront à le faire céder à la tentation. En effet, les médias, la multiplication des points de vente, la conception de vitrines et de rayons attrayants sont là pour le pousser à la consommation. L'information est omniprésente et prend comme support non seulement les radios, les télévisions, les affiches et les panneaux mais aussi les surfaces des moyens de transport (bus, voiture et métro) ou encore des bâtiments. En conséquence, la tentation est de plus en plus forte. Rajoutons à tout ce qui précède que l'identité et l'image sociale sont soutenues, dans nos sociétés, par le maintien d'un niveau de consommation. En effet, les membres de notre entourage (famille, voisins, collègues de travail, etc.) exercent sur nous des tensions pour que l'on consomme dans le but d'exister et d'être socialement reconnus. Désormais, comme le dit Frédéric Beigbeder dans $\mathrm{Au}$ secours, pardon (2007) : « Ce qu'on appelle la société de consommation devrait être : Société de Tentation ». Les logiques de consommation valent également pour les productions matérielles de type mobilier domestique.

\footnotetext{
${ }^{4}$ Comme par exemple : créer des points de ventes à proximité et de superficie de plus en plus grande, les facilités à travers les emprunts, les crédits bancaires ou encore les facilités de paiement.
} 


\section{LA CONSOMMATION DE MEUBLE DEPUIS 1988 EN MILLIARDS D'EUROS TTC}

$-10,0$
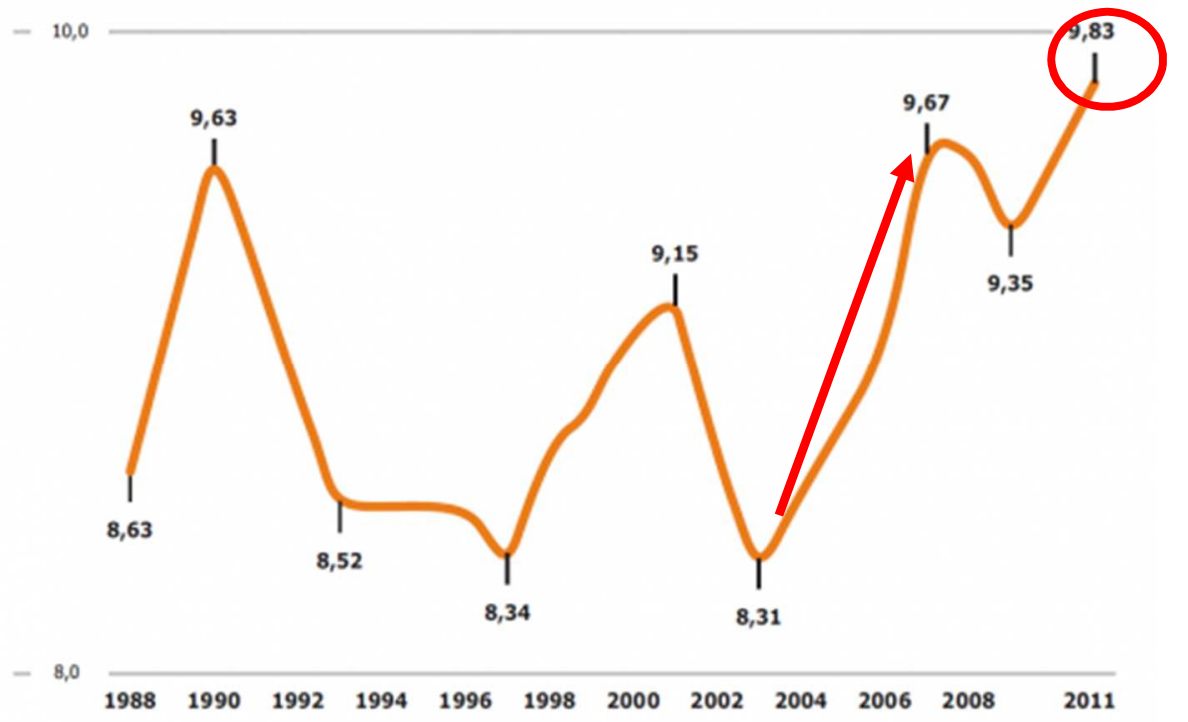

Source: IPEA

Figure 1 : La consommation de meubles en France de 1988 à 2011

La consommation de meubles en France a pris un élan progressif à partir de 2004 pour atteindre un pic de consommation en 2007 suivi d'une légère baisse en 2008 et en 2009 (Figure 1). Celle-ci semble être directement liée à la crise économique de cette période qui frappe tout particulièrement l'Europe et la France. La fin de l'année 2009 est marquée par un nouvel élan qui se poursuit en 2010. D'après le bilan de la consommation de meubles en France (2010), publié par l'Ipea ${ }^{5}$, le marché « a progressé de 2,7\% en valeur, à surfaces évolutives et en euros courants, pour atteindre 9,6 milliards d'euros TTC $»^{6}$. La progression continue au début de l'année 2011. En effet, d'après la Fnaem : « +5,9\%, c'est l'évolution du marché du meuble en cumul sur ce premier trimestre 2011

\footnotetext{
${ }^{5}$ En France, le secteur de l'ameublement fait l'objet d'un grand intérêt auprès des organisations telles que l'Ipea (Institut de promotion et d'études de l'ameublement). C'est un organisme d'études des professionnels de la filière, créé en 1979 par les industriels et les distributeurs français de l'ameublement. Cette association regroupe l'Unifa (Union nationale des industries françaises de l'ameublement) et la Fnaem (Fédération nationale de l'ameublement et de l'équipement de la maison). Elle est centrée sur l'étude de la production des meubles, de leur distribution, de leur consommation (marché) ainsi que l'étude du commerce extérieur.

${ }^{6}$ Bilan du marché de meubles domestiques (2010). Version PDF récupérée de l'adresse web : http://www.mobicite.com/index.php//Developpement/Economie/Le-marche-du-meuble-renoue-avec-la-croissance-en-2010
} 
et le meilleur démarrage enregistré depuis l'année 2007, année record qui s'était soldée par une progression en fin d'année de $+7,3 \% »^{7}$. A la fin de l'année 2011, le marché a enregistré son plus haut niveau depuis 1988 avec un chiffre d'affaires atteignant 9,83 milliards d'euros TTC $^{8}$. La Fédération française du négoce de l'ameublement et de l'équipement de la maison (Fnaem) a annoncé le jeudi $1^{\mathrm{er}}$ mars 2012 : « Le marché de la distribution de meubles poursuit sa progression, quasi constante depuis dix ans, et enregistre en 2011 un résultat record, à 9,83 milliards d'euros »'. Toutefois, producteurs et consommateurs sont-ils conscients des conséquences d'un tel rythme de production de meubles sur l'épuisement des ressources naturelles ? Le président de l'Ifa (Industries françaises de l'ameublement), a adressé une lettre aux dirigeants d'entreprises de fabrication d'éléments d'ameublement à usage domestique stipulant qu' :

"À compter du $1^{\mathrm{er}}$ janvier 2012, la loi "Grenelle 2" impose aux émetteurs sur le marché, à savoir les fabricants et les importateurs de meubles, d'assumer la gestion des éléments d'ameublement dont se débarrassent les particuliers et les entreprises... L'Unifa, en coopération avec la Fnaem et la Fmb (Fédération des magasins de bricolage), souhaite lancer une expérimentation de terrain afin de préciser les gisements de déchets d'éléments d'ameublement à collecter, les coûts de collecte, de transport et de traitement de ces déchets et donc le montant des contributions que chaque entreprise devra payer au futur écoorganisme... ${ }^{10} »$

Ceci démontre qu'il existe une mobilisation visant à responsabiliser les producteurs et les importateurs sur la gestion des déchets des consommateurs et des entreprises. Une telle mobilisation prépare et invite les fabricants à changer d'attitude et de vision pour le bien de l'environnement, problématique qui se trouve au centre des préoccupations non seulement des organisations écologiques mais aussi des chercheurs scientifiques s'interrogeant sur les comportements de consommation à venir. La question est de savoir à présent si les consommateurs sont prêts à avancer le devoir de conserver le capital environnemental au détriment de leur besoin de changer et leur plaisir à innover.

\section{Lassitude, changements du mobilier domestique et tendance de surconsommation}

Pendant de nombreux siècles, les ménages ont eu un usage durable du mobilier. Souvent le lit, l'armoire et la commode étaient transmis d'une génération à l'autre. Les changements étaient surtout d'origines fonctionnelles (meuble cassé ou bois usé). Actuellement, les individus semblent rapidement se lasser d'un meuble parce que celui-ci

\footnotetext{
${ }^{7}$ Evolution du marché (mise à jour 20 avril 2011). Récupérée du site : http://wwww.fnaem.fr/chiffres_cles.ffm

${ }^{8}$ Source : http://www.fnaem.fr/dl/communiques/FNAEM_COMMUNIQUE_113.pdf

${ }^{9}$ Source : http://consommation.blog.lemonde.fr/2012/03/01/malgre-la-crise-les-francais-renouvellent-leur-interieur-avant-linstauration-dune-nouvelle-taxe/

${ }^{10}$ Disponible sur l'adresse web : www.mobicite.com/content/../111967/.../nvitation_reunion21avril2011.doc
} 
ne correspondrait plus à l'identité du consommateur, parce que trop démodé. Les motivations qui poussent au changement du mobilier dans les intérieurs sont variées. Elles proviennent de facteurs d'ordre économique (politique de production, influence des médias, tendance stylistique et esthétique de plus en plus éphémère, système de la mode, vente en ligne, banalisation de l'achat à crédit, etc.), sociologique (jugement de la société sur l'image de soi, identité sociale ${ }^{11}$, influence et imitation d'autrui) et psychologique (lassitude, ennui, rejet, complexe de différence). Parmi ces facteurs, je me suis particulièrement intéressée à l'étude du sentiment de lassitude en tant que phénomène déclencheur du désir de changement (Figure 2).

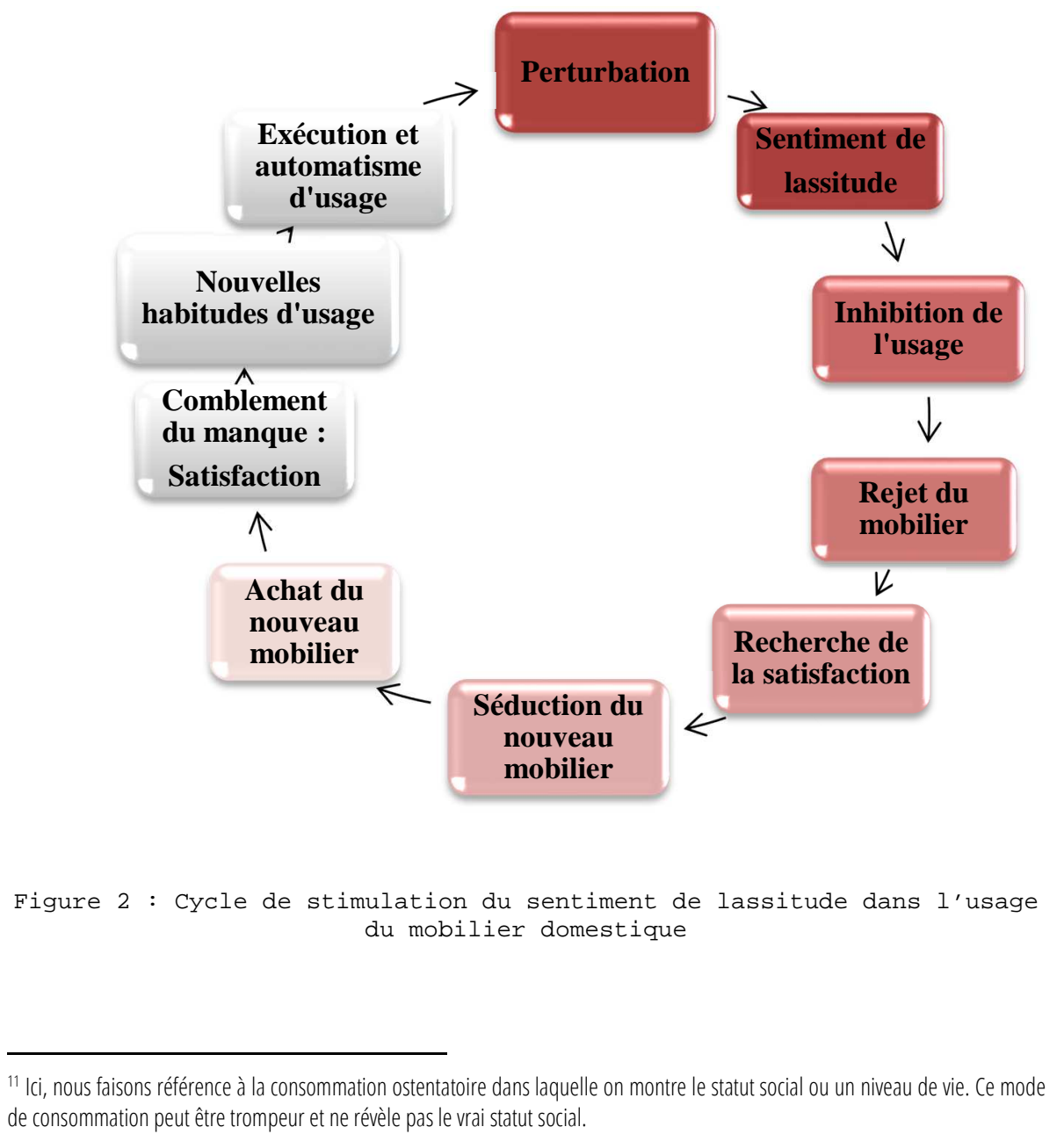

\footnotetext{
de consommation peut être trompeur et ne révèle pas le vrai statut social.
} 
Par lassitude, il faut comprendre ennui et fatigue morale suite à un usage monotone du même mobilier. Ce sentiment provoque le désir de changement et la quête du bienêtre moral. Le changement qui est « le passage d'un état à un autre ${ }^{12}$ est une éventuelle solution pour échapper à l'emprise de la lassitude. Il existe plusieurs moyens d'en produire, par exemple, par l'achat d'un nouveau meuble, par la récupération d'un meuble ancien ou encore par le re-bricolage de ce dernier. Dans cette étude, je me suis concentrée sur l'impact de la lassitude sur le comportement de consommation. Si l'on satisfait à chaque fois le désir de changement par un nouvel achat, la production de meubles augmente fatalement. Les conséquences de l'hyperconsommation se manifestent de plus en plus dans tous les domaines, y compris dans le secteur du mobilier, notamment par l'épuisement des ressources naturelles, la pollution ou encore la disparition des espèces. Le système dans lequel nous vivons souffre de ces abus. Toutes les phases de vie d'un mobilier, de la conception jusqu'au recyclage et au rejet devraient être impliquées pour la sauvegarde de l'environnement. Il semble que différentes mobilisations soient activées pour repenser notre consommation et adopter un comportement éthique. Le changement de mobilier n'est pas stimulé uniquement par des pulsions d'innovations permanentes guidées par la tendance. Actuellement, les discours écologistes font appel à l'inhibition d'une consommation de plus en plus « éphémère » en faveur d'une consommation écologiquement responsable. L'usager est alors invité à s'orienter vers des mobiliers plus sains et moins toxiques, construits à partir de matériaux écologiques, voire même recyclés et recyclables, préservant le bien de l'environnement dans toutes les phases de production, de diffusion et d'utilisation. Finalement, le consommateur se trouve tiraillé entre deux comportements divergents :

- Le désir de changement stimulé perpétuellement par la mode, les tendances et le sentiment de lassitude.

- Le changement de consommation pour des comportements écologiques et l'inhibition de la consommation abusive et inutile.

\section{Programme de recherche}

Pour étudier et comprendre les croyances et les tendances de comportement, j'ai choisi de mener une étude basée sur une enquête quantitative. L'échantillon se compose de 126 Français habitant à Paris et Lyon. Contrairement aux zones rurales, le nombre de magasins et de grandes surfaces de vente de mobilier est plus élevé dans ces grandes villes, ce qui offre aux citadins « une facilité d'accès » à la consommation. La tentation y semble plus grande et le désir de changer et d'innover y est nettement plus marqué. A priori, la décoration de l'espace domestique et le choix des meubles me semblaient deux activités gérées principalement par des femmes. Afin de vérifier cette assertion, j'ai intégré dans l'enquête à la fois des hommes et des femmes. De plus, pour mieux

${ }^{12}$ Changement (n.d.) : Dans Larousse. Récupéré du site : http://www.larousse.fr/dictionnaires/francais/changement 
distinguer les différences de comportement entre les générations, l'échantillon a été soumis à la variable de l'âge formée sur deux intervalles, $25 / 35$ ans et $45 / 65$ ans. Les participants sont donc classés selon quatre catégories :

- 45 femmes âgées entre 25 et 35 ans.

- 34 hommes âgés entre 25 et 35 ans.

- 24 femmes âgées entre 45 et 65 ans.

- 23 hommes âgés entre 45 et 65 ans.

Le questionnaire comporte 21 questions fermées et s'achève par une question ouverte. Les thèmes traités sont les suivants :

- Les facteurs qui motivent le changement et l'innovation du mobilier domestique : les facteurs personnels, les facteurs sociaux, la publicité, les émissions de décoration, les magazines, l'attirance des vitrines, les promotions et les baisses des prix, les signes d'usures, le déménagement, la rénovation, la lassitude, les soucis d'harmonie, etc.

- Les facteurs qui inhibent l'innovation du mobilier domestique : la stabilité et la sensation de confort avec les mobiliers existants, le comportement écologique et responsable, la situation financière qui ne permet pas l'achat de nouveaux mobiliers, etc.

- Le rapport entre les usagers et les aménagements durables des espaces domestiques : la manifestation du besoin de changement entre 1 et 5 ans, entre 5 et 10 ans, à partir de dix ans ou l'absence du besoin de changement.

- La fréquence de changement du mobilier domestique : tous les 3 à 5 ans, tous les 5 à 10 ans, tous les dix ans et plus, jamais.

- La réaction en présence du sentiment de lassitude et du désir de changement : l'achat précipité, l'achat raisonné, le réaménagement de l'espace intérieur, le bricolage, l'abstinence d'achat pour des raisons financières ou des raisons écologiques.

- Les différentes attitudes et positions par rapport aux changements fréquents du mobilier domestique : le changement fréquent perçu comme source de plaisir ou moyen d'être toujours à la page, ou encore comme perte d'argent ou cause de nuisance à l'environnement.

- L'étude des préoccupations écologiques des consommateurs : renseignements sur les origines des matières premières du mobilier et leur biodégradabilité, les toxines libérées dans le cycle de production ou au cours de l'usage, le recyclage du produit après le rejet.

- Le type de mobilier utilisé par les participantes (mobilier avec un design tendance ou mobilier durable de type écologique) et le type de mobilier vers lequel elles ont l'intention de s'orienter lors du prochain changement. 
L'intégration d'hommes dans l'enquête a été difficile car ils étaient, dans la plupart des cas, indifférents au thème étudié. Ceci confirme, dans une certaine mesure, que le secteur de l'ameublement intéresse davantage la gente féminine. Les résultats qui suivent concernent particulièrement celles qui ont participé à l'enquête.

\section{Analyses et résultats}

Le sentiment de lassitude est à entendre en tant que sentiment d'ennui, de découragement, de fatigue, d'épuisement ou encore de tristesse. L'apparition, la disparition et la réapparition de ce sentiment semblent être assez variables selon les individus de l'échantillon. L'étude tend à montrer que des facteurs tels que le genre, l'âge, la situation économique, la situation familiale, ou encore la situation socioculturelle sont à corréler à ce phénomène. Les résultats ont révélé que les jeunes personnes se lassent presque trois fois plus que les personnes âgées : 37\% de sentiment d'ennui et de lassitude pour les jeunes contre seulement $13 \%$ pour les plus âgés (figure 3).

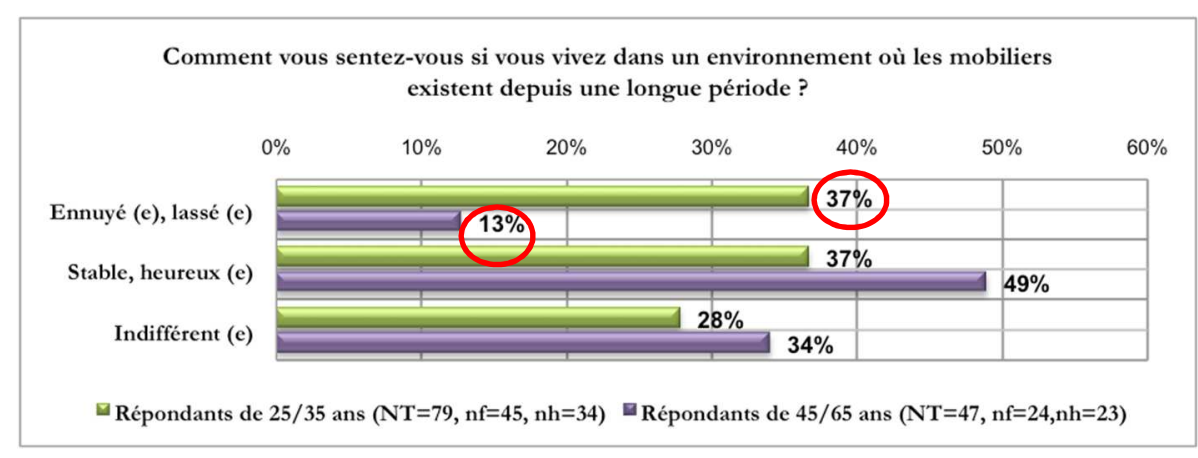

Figure 3 : Résultats en pourcentage des données collectées des deux générations sur la question de la durabilité de l'aménagement des mobiliers dans l'espace domestique

De plus, les résultats montrent, dans un premier temps, que les femmes se lassent plus que les hommes ( $42 \%$ des jeunes femmes se lassent de la stabilité des aménagements intérieurs contre $29 \%$ des jeunes hommes et $17 \%$ des femmes plus âgées contre $9 \%$ des hommes plus âgés) et que les jeunes femmes, dans un deuxième temps, se lassent plus que les femmes plus âgées (figure 4). Le désir de changement de mobilier serait une conséquence directe du ressenti de cette lassitude chez les sujets qui y sont sensibles. Leurs réactions face à ce sentiment peuvent également prendre d'autres formes que je détaillerai ultérieurement. 


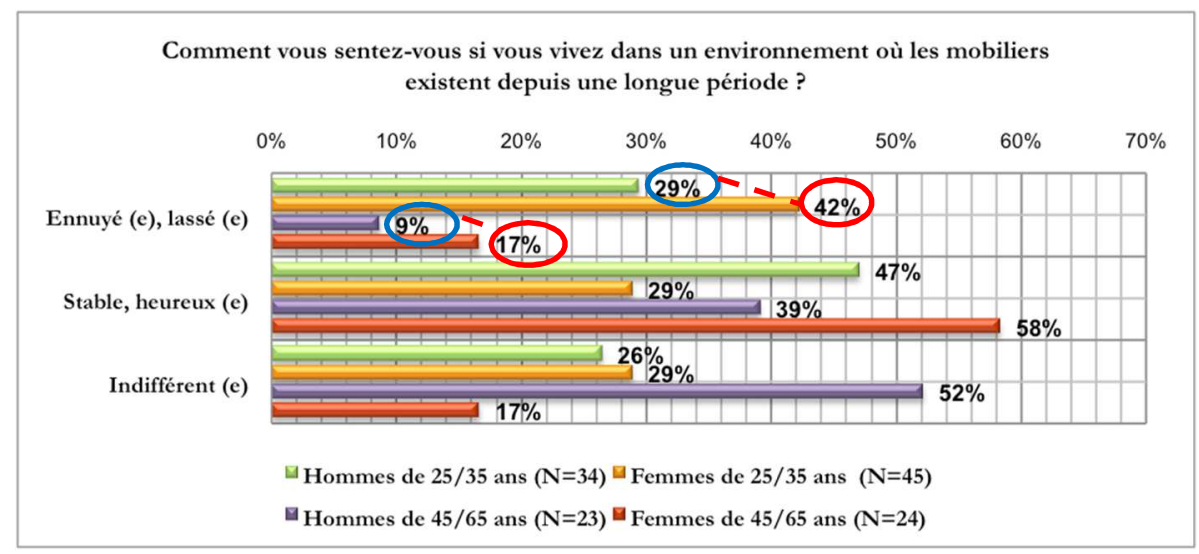

Figure 4 : Résultats en pourcentage des données collectées des quatre catégories sur la question de la durabilité de l'aménagement des mobiliers dans l'espace domestique

Le désir de changement n'est pas uniquement lié au sentiment de lassitude, mais également à celui de plaisir. La majorité des consommateurs ayant participé à cette enquête changent de mobilier pour se faire plaisir ou encore pour le plaisir de rajeunir. En effet, parmi les sujets âgés - et plus particulièrement les femmes - le sentiment d'être loin des années de jeunesse est d'autant plus fort qu'ils évoluent dans des environnements, qui, d'après eux, ont un style démodé. Dans ce cas, ils considèrent le changement comme une sorte de « relooking », une manière de rajeunir à travers l'image de l'intérieur domestique. Le désir de changement est nettement plus visible chez les jeunes femmes. Cette catégorie de population est celle qui réagit le plus rapidement face à l'ennui. En effet, 33\% des jeunes participantes (25/35 ans) déclarent désirer le changement dans les cinq premières années d'usage du mobilier, alors que chez les femmes plus âgées ( $45 / 65$ ans), ce désir est moins présent (4\%) et se manifeste entre 5 et 10 ans d'usage. Les femmes plus âgées semblent avoir une fréquence de changement du mobilier plus étendue dans le temps que les jeunes femmes (figure 5). 


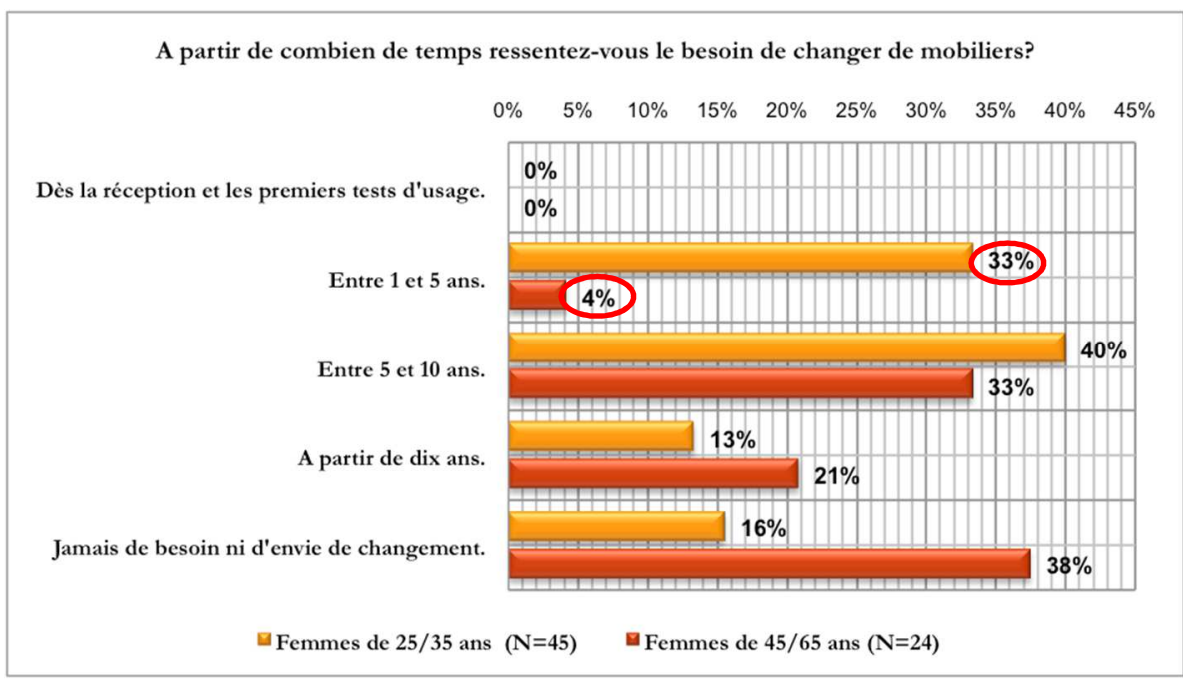
Figure 5 : Résultats en pourcentage des données collectées sur la question de la manifestation du besoin de changement de mobilier domestique

Par déduction, les jeunes femmes sont également celles qui passent le plus souvent à l'action. Leur fréquence de consommation s'avère être plus élevée que celle des femmes plus âgées. En effet, si l'on considère la fréquence de changement « tous les 3 à 5 ans ", on constate que les jeunes femmes changent de mobilier environ 3 fois $1 / 2$ plus que les femmes âgées (29\% contre seulement $8 \%$ ). Celles-ci passent au changement réel de mobilier à partir de 5 à 10 ans d'usage et surtout « tous les 10 ans et plus » (figure 6).

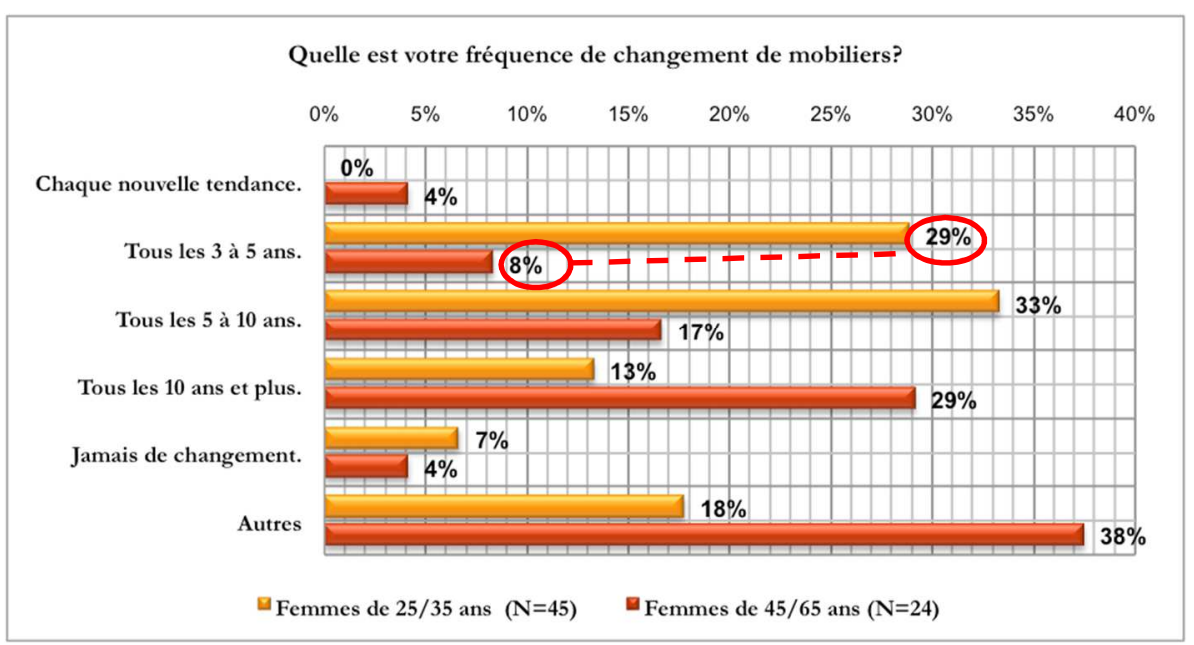


Figure 6 : Résultats en pourcentage des données collectées sur la question de la fréquence du changement de mobilier domestique

La réaction au phénomène de lassitude et au désir de changement peut prendre plusieurs formes. En effet, les participantes ${ }^{13}$ ont recours à des pratiques diverses en réponse à la lassitude. Elles consomment certes du nouveau mobilier (ce qui génère des impacts sur les plans économique et écologique), mais elles utilisent également d'autres moyens. D'une part, le déplacement des meubles entraîne l'illusion d'un changement d'intérieur ; cette pratique est adoptée par $42 \%$ des jeunes femmes et $46 \%$ des femmes plus âgées. D'autre part, le bricolage constitue une réaction contre l'ennui d'un environnement intérieur qui ne varie pas. Il s'agit alors de transformer les meubles en leur donnant un nouvel aspect esthétique ou encore une nouvelle vie quand il s'agit de la restauration des vieux meubles. Cette pratique est essentiellement adoptée par les femmes (36\% des jeunes femmes et 38\% des femmes plus âgées) ; $24 \%$ des jeunes hommes et $17 \%$ des hommes plus âgés bricolent, quant à eux, leur mobilier (figure 7).

\footnotetext{
${ }^{13}$ Cette partie de l'analyse concerne la deuxième et la troisième question de recherche. Je rappelle que ces questions s'adressent à la catégorie des personnes la plus touchée par le sentiment de lassitude quant à la durabilité des aménagements intérieurs. En me référant sur les résultats précédents, cette catégorie de personnes correspond aux femmes de l'échantillon.
} 


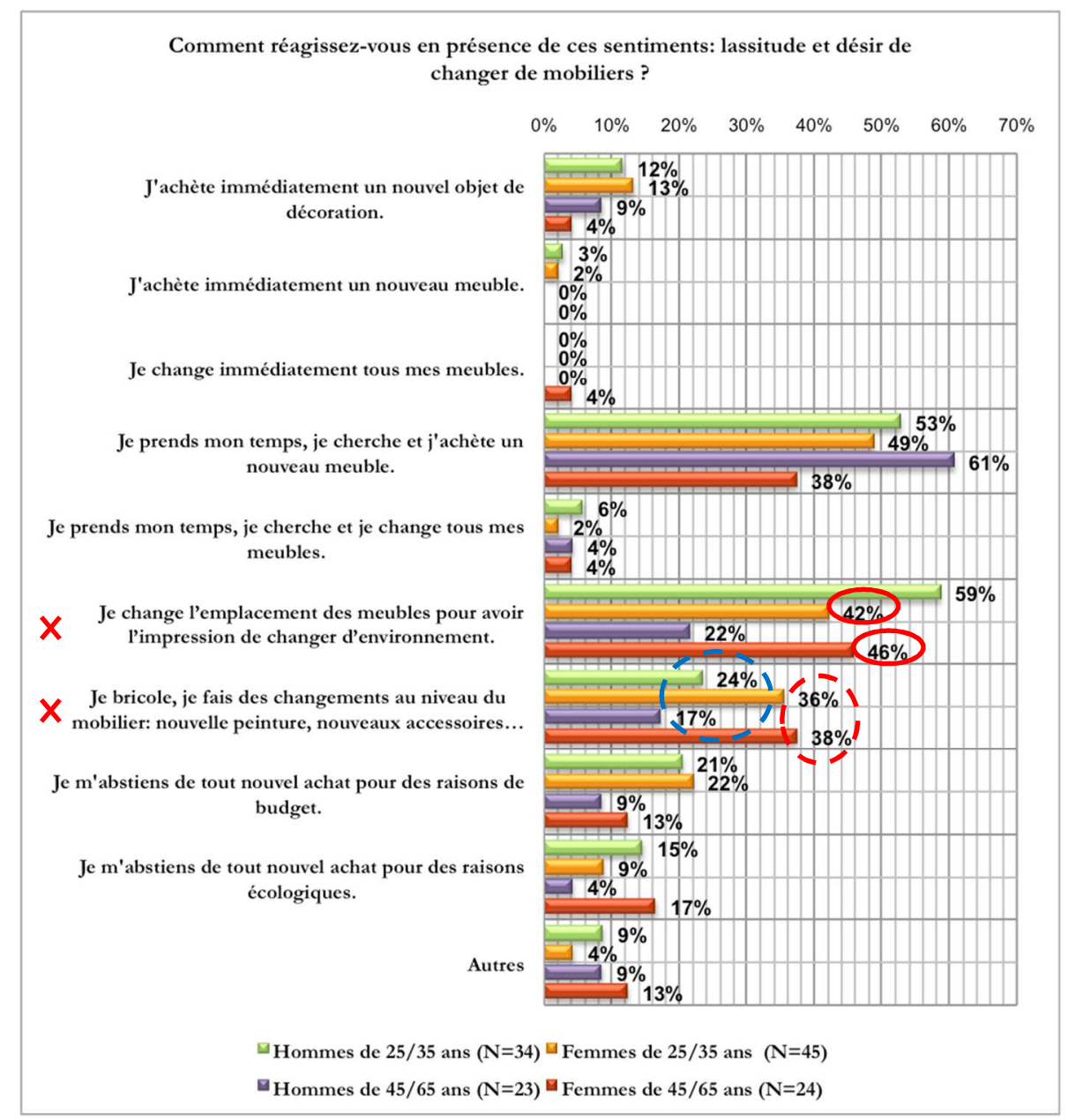

Figure 7 : Résultats en pourcentage des données collectées sur l'étude des réactions en présence des sentiments de lassitude et de désir de changement de mobilier domestique

Deux attitudes assez opposées semblent se dégager des réactions des participantes à la question des sentiments d'ennui et de lassitude vis-à-vis du mobilier domestique. Les jeunes femmes (25/35 ans) tendent à réagir plus rapidement que les femmes plus âgées (45/65 ans) et enregistrent, comme nous l'avons vu précédemment, la fréquence de changement la plus élevée (tous les 3 à 5 ans). Le comportement des jeunes femmes tend donc vers la surconsommation. Cependant, le questionnaire révèle que dans la plupart des cas, elles sont étudiantes ou en début de carrière et n'ont pas les moyens d'accéder à ce cycle de consommation. Elles se trouvent donc tiraillées entre leur sentiment de 
lassitude qui stimule leur désir d'innovation permanente et leur faible pouvoir d'achat. Elles développent donc d'autres types de réponses pour parer ce sentiment de lassitude. Elles optent par exemple plus facilement pour le réaménagement du mobilier ou pour le bricolage. Ces deux réponses qui constituent des formes de résistance à la tentation de consommation chez les jeunes femmes pourraient traduire un comportement en faveur de l'écologie. Sauf que celles-ci semblent adopter ce comportement plus en raison d'un manque de moyens que d'une réelle responsabilité écologique. D'ailleurs, $49 \%$ des jeunes participantes indiquent qu'elles n'ont pas les moyens financiers pour changer de mobilier (figure 8). Pour les femmes plus âgées, la situation est inversée : 58 \% des femmes plus âgées se sentent heureuses dans des environnements stables (figure 4). La moitié d'entre elles ne désirent pas de changement (figure 8). Cependant, certaines d'entre elles adhèrent au changement pour refléter une image plus jeune. En dépit de moyens financiers plus confortables, elles sont moins impliquées dans la tendance de surconsommation. En revanche, elles représentent, parmi les participants à l'enquête, la catégorie qui se soucie le plus des questions environnementales ${ }^{14}$, révélant ainsi un comportement écologique et responsable. En effet, $46 \%$ des femmes âgées signalent qu'elles réduisent leur consommation de mobilier domestique parce qu'elles sont conscientes de l'impact de la surconsommation sur l'environnement (figure 8).

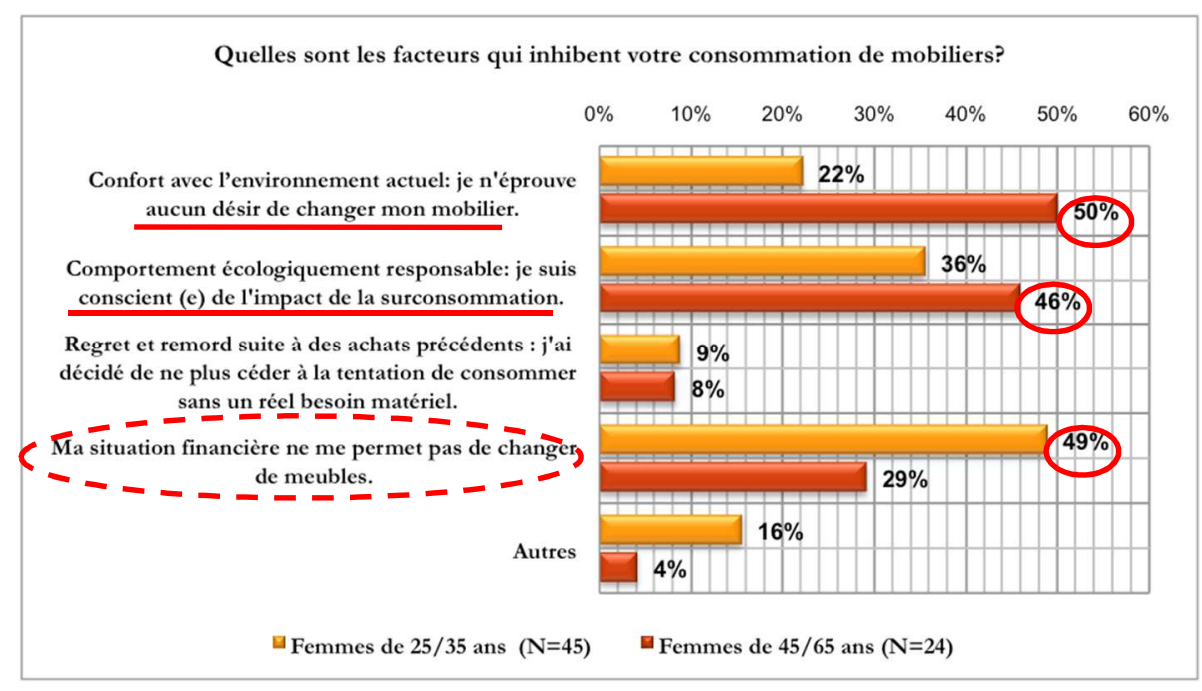

Figure 8 : Résultats en pourcentage des données collectées sur l'étude des facteurs qui inhibent la consommation de mobilier domestique

Concernant l'intérêt porté aux préoccupations écologiques, il apparaît que les participantes se renseignent davantage sur la provenance des matières premières du mobilier

${ }^{14}$ Ce point est détaillé dans l'analyse qui suit. 
que sur l'impact de sa consommation sur leur santé et sur l'environnement. Lors de l'achat, les questions sur les matériaux biodégradables, les toxines libérées dans le cycle de production ainsi qu'au cours de l'usage ou encore le recyclage n'éveillent que très peu d'intérêt. Les femmes âgées sont celles qui posent le plus de questions au sujet de l'écologie, ce qui permet d'éclairer leurs préoccupations sur ce thème, plus prononcées que celles des jeunes femmes (figures 9, 10,11,12 et 13).

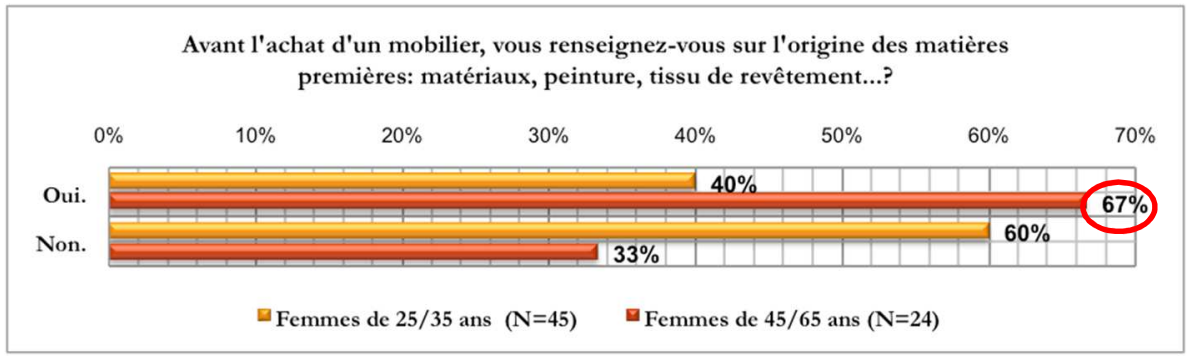

Figure 9 : Résultats en pourcentage des données collectées sur l'étude de $l^{\prime}$ intérêt porté aux préoccupations écologiques. Question 1 .

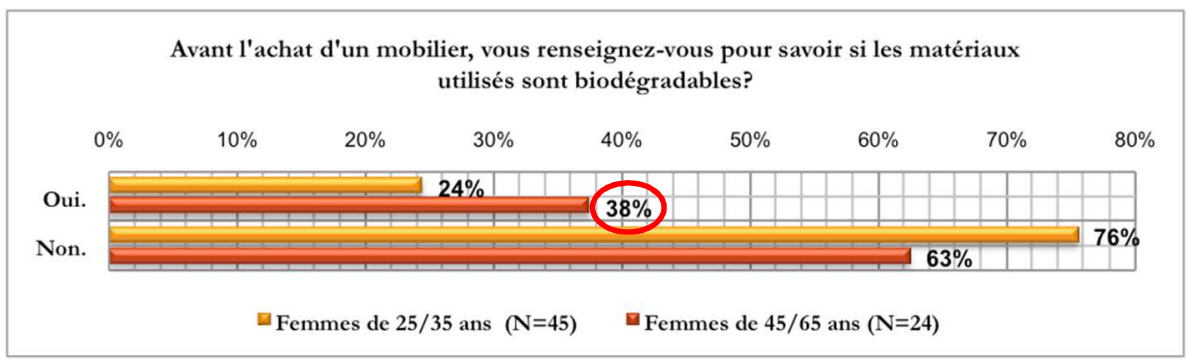

Figure 10 : Résultats en pourcentage des données collectées sur l'étude de $l^{\prime}$ intérêt porté aux préoccupations écologiques. Question 2 .

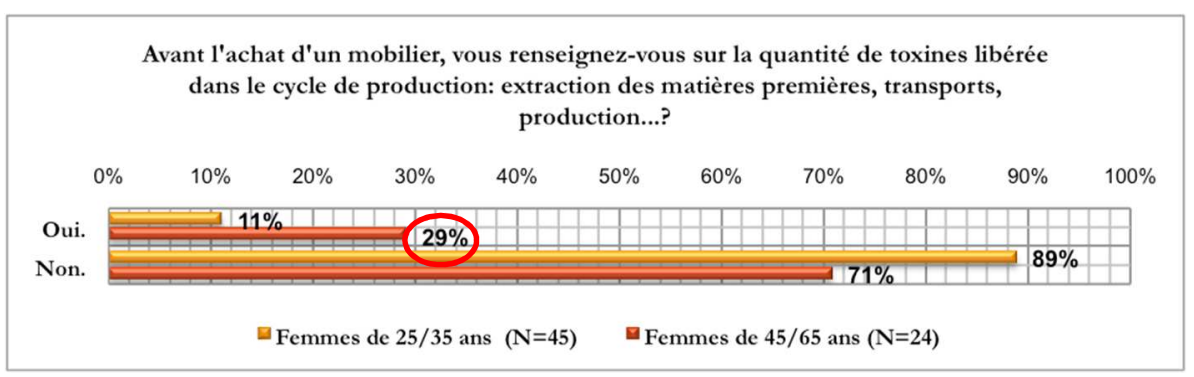


Figure 11 : Résultats en pourcentage des données collectées sur l'étude de l'intérêt porté aux préoccupations écologiques. Question 3.

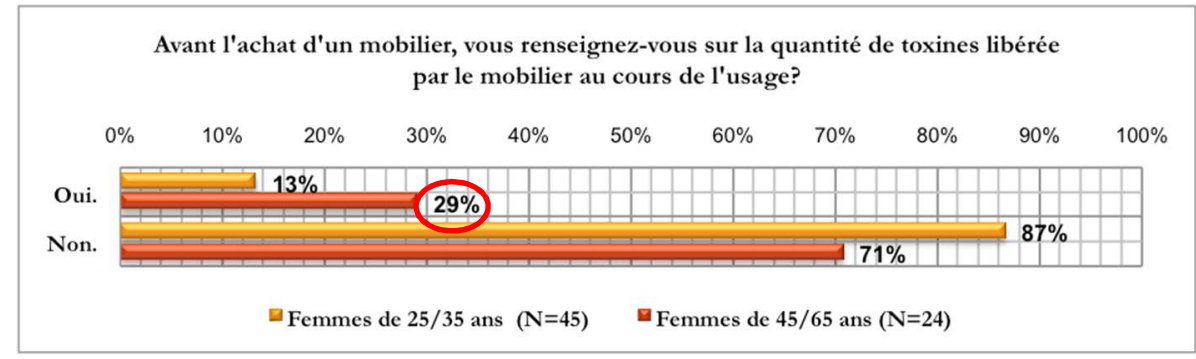

Figure 12 : Résultats en pourcentage des données collectées sur l'étude de l'intérêt porté aux préoccupations écologiques. Question 4.

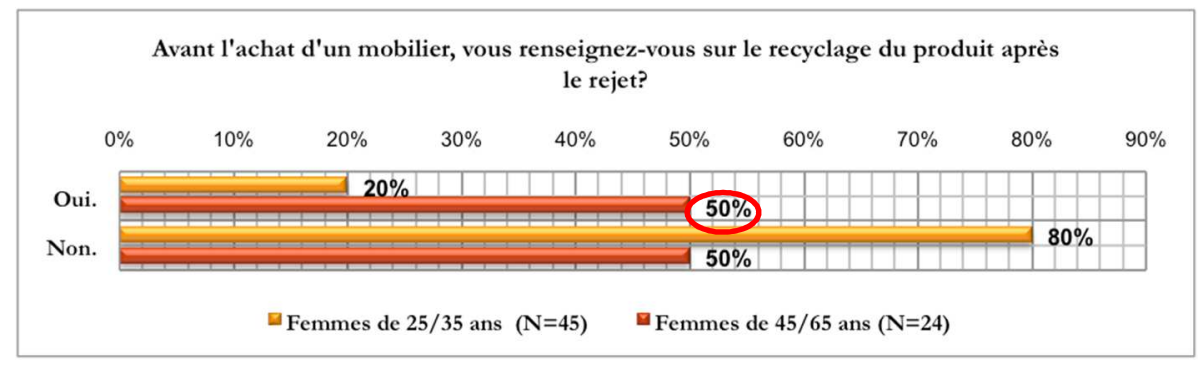

Figure 13 : Résultats en pourcentage des données collectées sur l'étude de l'intérêt porté aux préoccupations écologiques. Question 5.

D’une manière générale, qu'elles soient jeunes ou plus âgées, les participantes qui se soucient de la relation entre le secteur de l'ameublement et l'écologie et qui posent des questions à ce sujet avant l'achat d'un mobilier restent minoritaires par rapport aux femmes qui ne semblent pas s'y intéresser. Tandis que certaines personnes réclament beaucoup d'informations ou se plaignent même du manque d'informations à ce sujet, d'autres ne s'en préoccupent pas. Cette enquête a probablement éveillé la conscience de certaines femmes concernant le lien entre écologie et secteur de l'ameublement. Elle pourrait peut-être même, à terme, encourager certaines consommatrices à changer de type de mobilier lors des prochains achats en optant pour un mobilier de type écologique plutôt qu'un mobilier de type design, c'est-à-dire un mobilier susceptible de se démoder rapidement (plus particulièrement au niveau des propriétés esthétiques : couleur, matériaux, etc.). Ce type de mobilier est tout à fait susceptible d'accélérer le sentiment de lassitude et de rejet. Dans la majorité des cas, les préoccupations écologiques 
ne constituent pas une priorité prise en compte au moment de sa fabrication. Un mobilier de type écologique est justement conçu dans le respect de l'environnement et de la santé de l'usager, et ce de l'extraction des matières premières jusqu'au recyclage, ce qui n'est pas le cas pour le mobilier de type design. Or, il apparaît dans les résultats de l'enquête que la majorité des participantes possèdent plus de meubles de type design ( $51 \%$ des jeunes femmes et $50 \%$ des femmes plus âgées) que de meubles durables de type écologique (16\% des jeunes femmes et 17\% des femmes plus âgées) (figure 14).

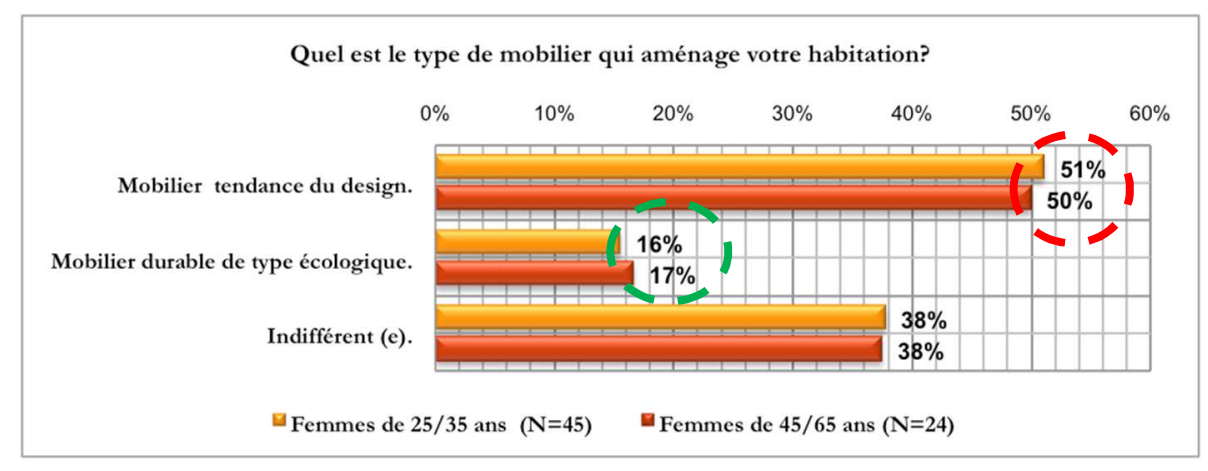

Figure 14 : Résultats en pourcentage des données collectées sur la question du type de mobilier en cours d'usage

En termes de prévisions de consommation de mobilier, il semblerait que le marché du mobilier de type design ait des chances de garder une certaine stabilité au cours des prochaines années. En effet, il y a autant de femmes qui projettent d'acheter ce type de meubles «tendance » (49\% des jeunes femmes et 50\% des femmes plus âgées) (figure 15) que de femmes, qui, actuellement, utilisent ce type de mobilier ( $51 \%$ des jeunes femmes et $50 \%$ des femmes plus âgées) (figure 14). Toutefois, d'autres indicateurs montrent que le marché du mobilier de type écologique va probablement doubler. En effet, le nombre de femmes ayant l'intention d'acheter des meubles durables est en augmentation. Il apparaît que 36\% des jeunes femmes et 33\% des femmes plus âgées ont l'intention d'acheter des mobiliers de type écologique contre $16 \%$ des jeunes femmes et $17 \%$ des femmes plus âgées qui en possèdent déjà au moment de l'enquête (figures 14 et 15). Les meubles écologiques tendent à concurrencer les meubles « tendance » en attirant de plus en plus de consommatrices. 


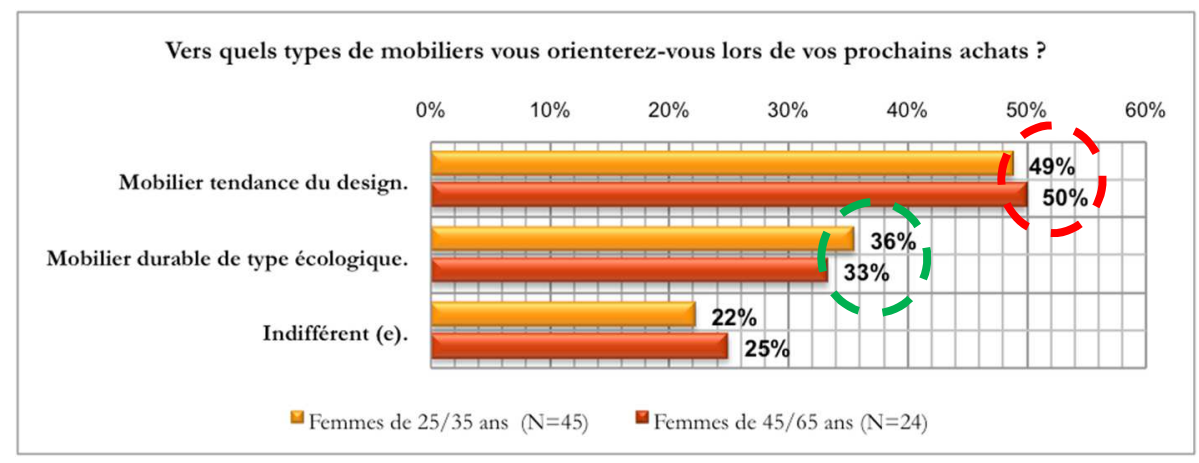

Figure 15 : Résultats en pourcentage des données collectées sur la question du type de mobilier dans les prochains achats

\section{Conclusion}

Comme d'autres secteurs de consommation (alimentation, transport, habillement, etc.), le secteur du mobilier domestique est soumis aux phénomènes de la mode et de l'éphémère. Il est, en ce sens, susceptible d'être associé aux sentiments d'ennui, de lassitude et de routine qui stimulent le désir de changement et d'innovation auprès du consommateur. D’après les résultats de cette enquête, les femmes sont particulièrement sensibles à ces phénomènes et ces sentiments auxquels elles réagissent de manières différentes. Qu'elles soient jeunes ou plus âgées, la majorité des participantes ont tendance à adopter des comportements qui respectent l'environnement. Il apparaît aussi que la majorité des participantes plus âgées est plus consciente de leur comportement écologique que la plupart des plus jeunes. L'ennui, la lassitude et la routine sont des sentiments qui suivent une boucle fermée, continuellement activée par des agents perturbateurs tels que les médias, les leaders d'opinion, l'image de soi, etc. L'implication particulière des femmes dans ce secteur et dans cette boucle mériterait que l'on se penche davantage sur la nature et les origines de ces sentiments. Sont-ils des phénomènes naturels ou culturels ? À quel moment de l'histoire sont-ils apparus dans le secteur de l'ameublement, impliquant celui-ci dans le phénomène de la mode et la tendance à la surconsommation ? Pourquoi les femmes sont-elles plus concernées par le besoin de renouvellement ? La manifestation de la lassitude a-t-elle un lien avec le temps qu'elles passent chez elles ? Sont-elles la cible d'un marketing particulier ? Que peuvent apporter les femmes les plus intéressées par ce secteur et les plus sensibles aux sentiments d'ennui et de lassitude au design écologique des mobiliers domestiques ? En ce qui concerne les préoccupations écologiques, c'est l'ensemble du processus de conception, de production, de diffusion, de sensibilisation et d'information qu'il faudrait revoir. D'une manière générale, le consommateur conscient, averti, avisé ou informé devrait profiter de sa position déterminante dans le marché de l'ameublement pour 
agir et réclamer plus de renseignements et plus de considérations pour l'équilibre de l'environnement, pour sa santé et celle des générations futures.

\section{Bibliographie}

Baudrillard J. (1970), La société de consommation. Paris : Denoêl.

Beigbeder F. (2007), Au secours, pardon. Paris : Grasset. 